\title{
Microscopy Method for Characterization Oil Uptake in Fried Foods
}

\author{
David M. Williams ${ }^{1}$, Jing Guo ${ }^{2}$ and Robert Fletcher ${ }^{2}$
}

1. Analytical Sciences, The Dow Chemical Company, Midland, MI USA

2. Food \& Nutrition R\&D, The Dow Chemical Company, Midland, MI USA

The Dow Chemical Company is an industry leader in providing state-of-the-art products and technical service to the food industry. A microscopy method was needed to help evaluate the efficacy of WELLENCE ${ }^{\mathrm{TM}}$ Fat Reduction based batter coating formulations to inhibit oil uptake in french fries during cooking.

Osmium tetroxide and Oil Red O staining methods ${ }^{[1]}$ were adapted. Optical microscopy demonstrated these methods successfully stained cooking oil in cross sections of uncoated and WELLENCE Fat Reduction batter coated french fries. Sections measuring approximately 4-5 $\mathrm{mm}$ thick of the cooked uncoated and coated french fries were paced in $1 \%$ aqueous osmium tetroxide for 5-minutes then rinsed in running tap water for 20 -minutes. Additional sections were stained in $0.5 \%$ Oil Red O (in propylene glycol) for 30-minutes, differentiated in $85 \%$ propylene glycol and then rinsed in water. All stained sections were submerged in DI water in a small petri dish and imaged using coaxial illumination. Each sample was then mounted on sample stubs using a cyanoacrylate adhesive and sectioned 150 um-250 um thick using a Series 1000 Vibratome vibrating microtome. The Vibratome sample trough was filled with distilled water to facilitate the sectioning and easy removal of the cut sections. The cut sections were mounted on glass slides and imaged.

Using optical microscopy it was observed that osmium stained the cooking oil black as well as lightly staining the potato cell membranes of the uncoated sample (Figure 1). The Oil Red O stained the cooking oil reddish orange. The potato itself was not stained by oil Red $\mathrm{O}$ and remained white in color (Figure 2). Both stains showed a clear distribution of the cooking oil. The uncoated samples displayed significantly more oil staining throughout the french fry cross sections. Osmium staining also showed significant cellular damage from the loss of moisture within the french fry and it's replacement with oil. Very little penetration of the cooking oil into the interior of the coated french fries was observed. The hypothesis is that WELLENCE Fat Reduction in the batter formulation thermo-gels and forms a protective barrier keeping oil from penetrating the french fries.

Supporting the microscopy results is a quantitative analysis of the oil content of the coated and uncoated French fries using the Soxhlet ${ }^{[2]}$ extraction method.

References:

[1] Methods for Fats and Lipids, Chapter 9, page 140-141 and 143-144. Manual of Histologic Staining Methods of the Armed Forces Institute of Pathology. Third Edition, 1968. Library of Congress Catalog Card Number: 68-28409.

[2] Official Methods of Analysis. Association of Analytical Chemists, 1995, Washington, DC.

TM Trademark of The Dow Chemical Company. 

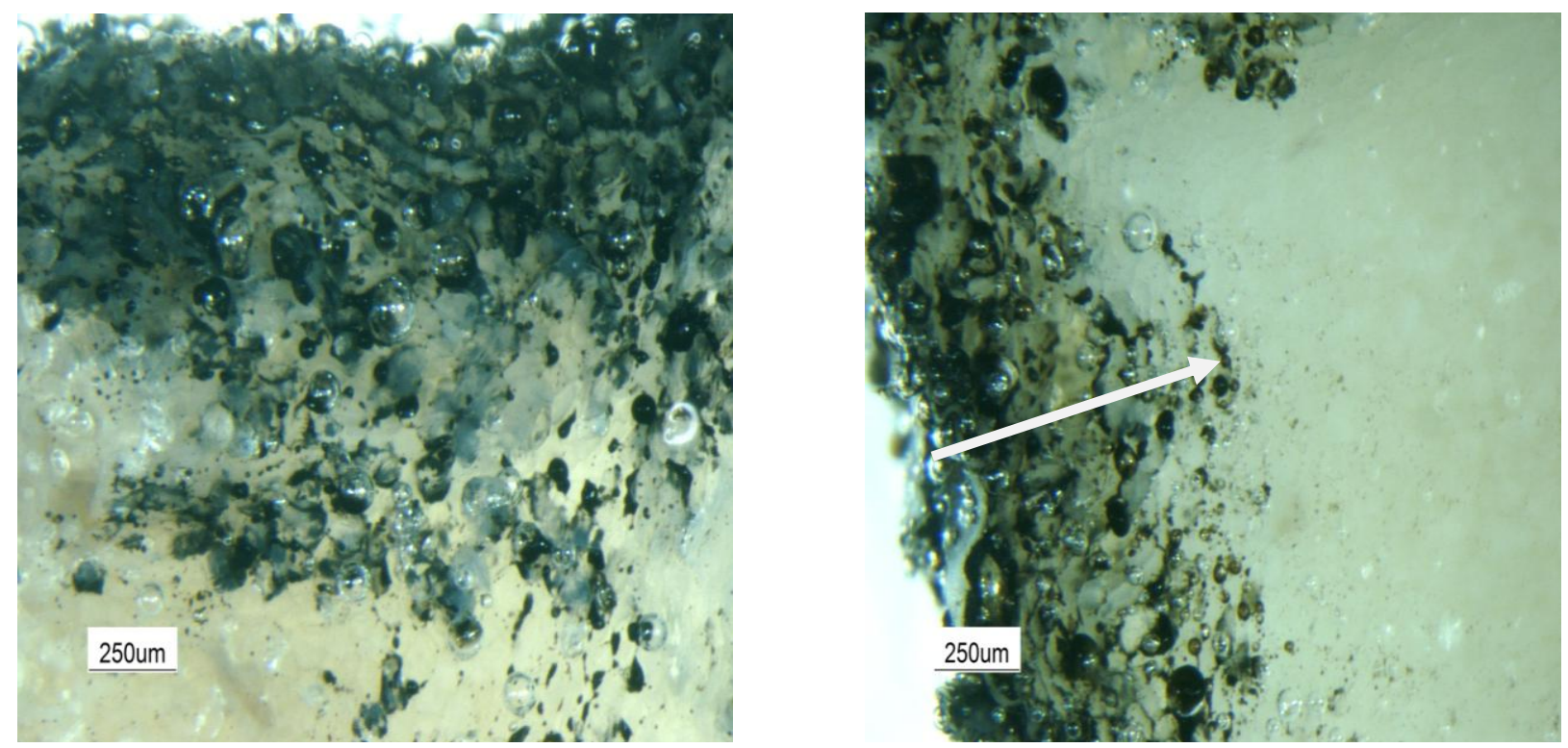

Figure 1. Osmium tetroxide stained samples. Uncoated french fry on left. WELLENCE ${ }^{\text {TM }}$ Fat Reduction batter coated French fry on right. Arrow indicates limit of oil penetration.
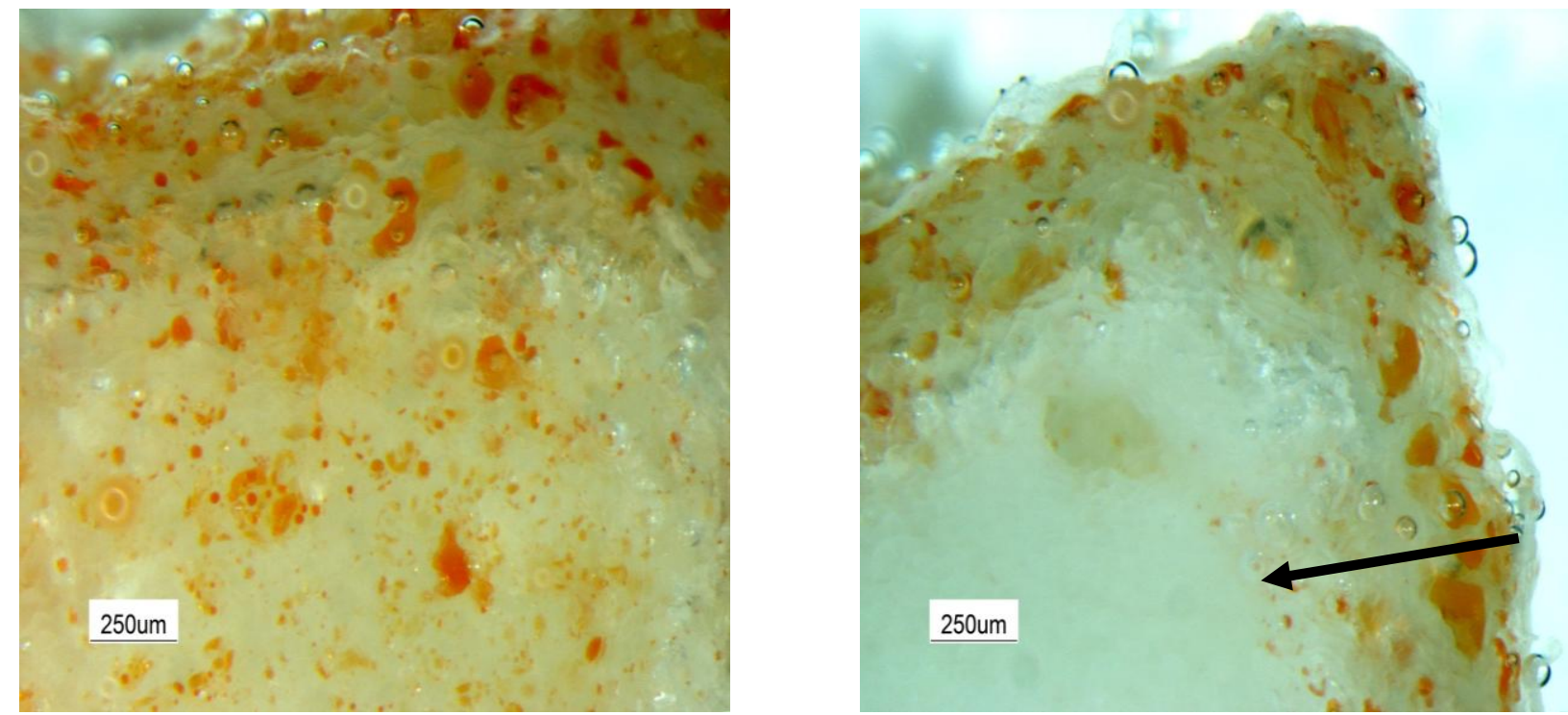

Figure 2. Oil Red O stained samples. Uncoated French fry on left. WELLENCE Fat Reduction batter coated French fry on right. Arrow indicates limit of oil penetration. 